\title{
Erratum: The Oral Bioavailability and Pharmacokinetics of Soluble and Resin-Bound Forms of Amphetamine and Phentermine in Man
}

\author{
Orville N. Hinsvark, Aldo P. Truant, Donald J. Jenden,
} and Joseph A. Steinborn

In this paper (Vol. 1, No. 4, 1973, pp. 319-328), the caption of Fig. 2 should read: Bioavailability of phentermine (circles) and amphetamine (squares) as resinates (filled symbols) and soluble salts (open symbols) expressed as the average time interval (ordinate) following a single dose for which the blood concentration exceeded a given threshold (abscissa). 